\title{
Prevalence of Folate and Vitamin B12 Deficiency Aged 1 to 6 Years
}

\author{
Dr. Jagdish Dubal \\ Assistant Professor, Department of Paediatrics, Gujarat Adani Institute of Medical Science, Bhuj, Gujarat, India
}

\begin{abstract}
Objective: To describe the distribution of folate and vitamin B12 deficiency in children. Materials and Methods: Folate and vitamin B12 serum concentrations were measured in sample of 1000 children. Adjusted prevalence, mean concentrations and relevant associations were calculated. Results: The overall prevalence of folate and vitamin B12 deficiency were $5.2 \%$ and $9.5 \%$, respectively. The highest prevalence of folate was found in the 2-year-old, and of vitamin B12 in the 1 year-old (9.1\%) groups. Conclusion: We describe the magnitude of folate and vitamin B12 deficiencies in children. The deficiency of both vitamins in children under 2 years old is a moderate public health problem in Mexico.
\end{abstract}

Keywords: Vitamin B12 deficiency, Folate Deficiency, Children

\section{Introduction}

Deficiency of micronutrients is still one of the most frequent nutritional problems worldwide. The lack of access to a varied diet including animal foods, grains, fruits, and vegetables, is the recurrent cause of deficiency, given those are the most important sources of micronutrients. ${ }^{1}$

Deficiencies of folic acid and vitamin B12 are known to cause megaloblasticanaemia, an anaemia which is characterized by presence of abnormally large erythrocyte precursor cells, megaloblasts, in the bone marrow and macrocytic red cells in the peripheral blood. ${ }^{2}$ These megaloblasts arise because of impaired DNA synthesis followed by ineffective erythropoiesis. nutrient required for maintenance of normal erytrhropoiesis, nucleoprotein and myelin synthesis, cell reproduction and normal growth. Vitamin B12 is a necessary cofactor in the methionine synthase reaction, which converts homocysteine into methionine. Deficiency of vitamin B12 could elevate plasma homocysteine which is a risk factor for cardiovascular diseases. ${ }^{3}$ Methionine is the required precursor for the formation of $\mathrm{S}$-adenosylmethionine, a universal methyl donor essential for methylation of phospholipids, neurotransmitters, amines, DNA, RNA and myelin basic protein. ${ }^{4}$ A reduction in this important methyl donor causes impaired DNA methylation that may contribute to altered fetal metabolic programming and increased risk for chronic diseases later in life. In the mitochondria vitamin B12 is required as a cofactor for the conversion of methylmalonylCoA to succinyl-CoA by the enzyme methylmalonyl-CoA mutase. In B12 deficiency, the concentration of methylmalonyl $\mathrm{CoA}$ is elevated and forms the by-product methylmalonic acid (MMA). Thus vitamin B12 insufficiency may influence carbohydrate and lipid metabolic pathways. ${ }^{5}$

Some studies have measured the magnitude of vitamin B12 deficiency in children under 5 years old, those had reported prevalence of $8 \%$ in six rural communities at central region in Mexico. Others reported prevalence of $30 \%$ in a subsample of data from the National Nutrition Survey 1999. In both cases, these studies do not represent the national population. The aim of this analysis is to describe the magnitude and distribution of folate and vitamin B12 deficiency in children aged 1 to 6 years, and to identify some associated sociodemographic and dietary determinants using data derived from the National Health and Nutrition Survey 2006 (ENSANUT-2006). Data from this study could help to plan public health strategies and policies focused on the control and reduction of these micronutrient deficiencies.

\section{Material \& Method}

The information collected includes socio-demographic, health and nutrition conditions; anthropometry and food consumption in all participants, included information of 1000 children aged 1 to 6 years. Blood samples were obtained from children to document the vitamins deficiencies. Statistical analysis and model based estimators were carried out to assess a possible selection bias, using reference variables like height, weight and the socioeconomic index. Informed consent was signed by the parents and ethical certificate was taken from the hospital committee.

Blood samples were drawn from the antecubital vein and collected in evacuated tubes. Blood was centrifugated in situ at $2500 \mathrm{~g}$ in a portable centrifuge. Serum was stored in cryotubes covered with aluminum foil to preserve them from light and kept in liquid nitrogen to be transported to the Nutrition laboratory. Laboratory methods Both serum folate and vitamin B12 were released from proteins using sodium hydroxide, dithiothreitol, and potassium cyanide, and transformed into cyanocobalamin and stable folates. Their concentrations were measured by competitive enzymatic immunoassays. The analysis was carried out using the SPSS V 15.0 software.

\section{Results}

The overall mean of serum folate concentration was 14.5 $\mathrm{ng} / \mathrm{mL}$ and the overall prevalence of folate deficiency was $5.2 \%$ (Table I). The highest prevalence was in children aged 1 to 2 years and it decreased with age down to in children aged 6 years. Prevalence of folate deficiency was higher in boys than in girls; in urban than in rural, and in the Southern than in the Northern region. Folate deficiency was also

\section{Volume 4 Issue 12, December 2015}




\section{International Journal of Science and Research (IJSR) \\ ISSN (Online): 2319-7064}

Index Copernicus Value (2013): 6.14 | Impact Factor (2014): 5.611

higher in indigenous children $(8.5 \%)$ compared with the national prevalence $(7.2 \%)$. However, none of these differences were significant.

The overall mean concentration of vitamin B12 was 550 $\mathrm{pg} / \mathrm{mL}$ and overall prevalence deficiency was $9.5 \%$. The prevalence of vitamin B12 deficiency varied in a nonsystematic manner between $8.5 \%$ in children aged 3 years and $13.6 \%$ in those aged 6 years.

\section{Discussion}

Folate deficiency is related mainly to a low intake of green leafy vegetables and legumes and meat. This deficiency is associated with megaloblastic anemia. During pregnancy, it is linked to an increased risk of low birth weight and neural tube defects and it is also associated with stunting. ${ }^{7}$ Vitamin B12 deficiency is more frequent in population with a poor or inadequate diet of animal foods. Other causes of this deficiency include: intestinal parasitosis in low-income population. Helycobacter pylori infections and atrophic gastritis in older adults (due to lack of intrinsic factor production). Strict vegetarian diets may be associated with vitamin B12 deficiency, both, in the mother and in the newborn, because the demand for this vitamin increases during pregnancy and lactation. Clinical manifestations of vitamin B12 deficiency include megaloblastic anemia which is undistinguishable from that produced by folate deficiency. Vitamin B12 is also crucially involved in the proliferation, maturation, and regeneration of neural cells therefore; its deficiency causes memory loss, dementia, and depression as well as cardiovascular disease and cerebrovascular ischemia. $^{8}$

We describe herein the magnitude and distribution of the prevalence of folate and vitamin B12 deficiency in a population sample of children younger than 6 years. The national overall prevalence of folate deficiency was relatively low, although the groups of 1 and 2 year-old children had the highest prevalence. While the national prevalence of vitamin B12 was always above 5\%. Such prevalence, close to $5 \%$, should be considered as a public health problem according to the WHO Technical Consultation on folate and vitamin B12. Results also indicate that the highest prevalence of folate and vitamin B12 deficiency have seen in the most vulnerable populations. Studies have shown an association of neuropsychiatric disorders with vitamin B12 deficiency, especially in the absence of anaemia or macrocytosis, therefore, an early screening for vitamin B12 has a merit, keeping in view the very high costs of late treatment of irreversible neuropsychiatric disorders arising from vitamin B12 deficiency.

This study contributes to generate information on the magnitude of the deficiency of both vitamins, since there are very few available publications documenting folate and vitamin B12 deficiencies in children at preschool age, one of the most affected group. ${ }^{9}$ The current strategies for reducing and controlling micronutrient deficiencies should thus be reexamined. Particular attention must be paid to the content of folic acid and vitamin B12 of the food or supplements distributed by food assistance programs like Oportunidades and Liconsa. ${ }^{8}$

\section{Conclusion}

We describe the magnitude of folate and vitamin B12 deficiencies in children. The deficiency of both vitamins in children under 2 years old is a moderate public health problem. There is an urgent need to advance our knowledge in a population specific manner to further improve the current guidelines of nutritional interventions for pregnant and lactating women that has direct relevance to child health.

\section{References}

[1] Musaiger AO, Hassan AS, Obeid O: The paradox of nutrition-related diseases in the Arab countries: the need for action. International journal of environmental research and public health 2011, 8:3637-71.

[2] Parry T: The diagnosis of megaloblastic anaemia. Clinical \& Laboratory Haematology 1980, 2:89-109.

[3] Pancharuniti N, Lewis CA, Sauberlich HE, Perkins LL, Go R, Alvarez J, Macaluso M, Acton R, Copeland R, Cousins A: Plasma homocyst (e) ine, folate, and vitamin B-12 concentrations and risk for early-onset coronary artery disease. The American journal of clinical nutrition 1994, 59:940-8.

[4] Schwartz DE, Vermeulen NM, O'day CL: S-adenosyl methionine regulation of metabolic pathways and its use in diagnosis and therapy. Google Patents, 2000.

[5] Siddiqua TJ, Allen LH, Raqib R, Ahmed T: Vitamin B12 Deficiency in Pregnancy and Lactation: Is there a Need for Pre and Post-natal Supplementation? Journal of Nutritional Disorders \& Therapy 2014, 2014.

[6] Scrimshaw NS: Articles based on an international workshop on iron-deficiency anaemia Editorial introduction. Food and Nutrition Bulletin 1996, 17.

[7] Safi J, Joyeux L, Chalouhi G: Periconceptional folate deficiency and implications in neural tube defects. Journal of pregnancy 2012, 2012.

[8] Cuevas-Nasu L, Mundo-Rosas V, Shamah-Levy T, Méndez-Gómez Humaran I, Ávila-Arcos MA, RebollarCampos M, Villalpando S: Distribución de la prevalencia de deficiencia de folato y vitamina B12 en niños mexicanos de 1 a 6 años de edad. Encuesta con base poblacional. Salud Pública de México 2012, 54:116-24.

[9] Elmahdi TE, Alzahrani SS, Al-hazmi A, Mohammed NE: Assessment of Nutritional Status in Male School Children using Complete Blood Count and Serum Folic Acid. Int $\mathbf{J}$ of Multidisciplinary and Current research 2015, 3. 